\author{
Dennis J. Ternet \\ Ronald G. Larson \\ L. Gary Leal
}

\section{Flow-aligning and tumbling in small-molecule liquid crystals: pure components and mixtures}

Received: 7 December 1998

Accepted: 10 March 1999
D.J. Ternet $(\varangle) \cdot$ L.G. Leal

Department of Chemical Engineering

University of California, Santa Barbara

Santa Barbara, CA 93106, USA

R.G. Larson

Department of Chemical Engineering

University of Michigan, Ann Arbor,

MI 48109, USA

\begin{abstract}
The results of an experimental study to measure the tumbling parameter, $\lambda$, for various small-molecule liquid crystals and their mixtures are presented. The methods used include textural observations (twist walls), a direct method, a rheological method, and the oscillatory method developed by Mather, Pearson, and Burghardt in 1995. The single-component results are compared with a molecular theory derived in 1995 by Archer and Larson as well as Kröger and Sellers, which predicts the temperature dependence of $\lambda$,
\end{abstract}

while the results from the binary mixtures are compared to a continuum theory derived by Rey in 1996, giving the concentration dependence of $\lambda$. The results from the four experimental methods agree with each other for single-component liquid crystals, but not for mixtures. This suggests a failure of the single director Leslie-Ericksen theory to describe the rheology of liquid crystal mixtures.

Key words Liquid crystal - Conoscopy $\cdot$ Flow-aligning $\cdot$ Tumbling $\cdot$ Mixtures

\section{Introduction}

The behavior of a small-molecule or polymeric nematic in a simple shearing flow depends strongly on whether the nematic is of the tumbling or flow-aligning type. According to the Leslie-Ericksen continuum theory, which assumes a nematic with a single director, tumbling behavior occurs when the ratio $\alpha_{3} / \alpha_{2}$ of Leslie viscosities is negative, while the nematic is flow-aligning if $\alpha_{3} / \alpha_{2}>0$. For a flow-aligning nematic, the director (a unit vector parallel to the average molecular orientation) in a shearing flow is driven by viscous torques towards a flow-alignment angle,

$\theta_{\mathrm{fa}}=\frac{1}{2} \operatorname{acos}(1 / \lambda)$,

where $\theta_{\mathrm{fa}}$ is the angle that the director makes with respect to the flow direction and $\lambda$ is the so-called reactive or tumbling parameter defined in terms of the Leslie viscosities by: $\lambda=\frac{1+\alpha_{3} / \alpha_{2}}{1-\alpha_{3} / \alpha_{2}}$.

For a flow-aligning nematic $\left(\alpha_{3} / \alpha_{2}>0\right), \lambda$ is greater than unity, while for a tumbler, $\lambda<1$.

For a tumbling nematic, the steady solution no longer exists, and in this case hydrodynamic torques act to continuously rotate the director in a direction consistent with the vorticity of the flow. A variety of instabilities typically ensue, leading to various textures such as bands, stripes, roll cells, "thread" and "worm" textures, producing an irregular, disclination-filled pattern known as director turbulence (Pieranski and Guyon 1974; Manneville 1976, 1981; Carlsson 1984; Zuñiga and Leslie 1989; Larson 1993).

Because of the large effect it has on the behavior of a nematic in a shearing flow, one would like to be able to predict the value of $\lambda$ from a molecular theory, and especially to predict whether it is greater than or less than unity. For polymeric nematics composed of rod- 
like molecules in a solvent, molecular theories predict tumbling behavior at lower shear rates (Kuzuu and Doi 1983, 1984). This prediction has been confirmed by experimental studies on nematic polymer solutions (Burghardt and Fuller 1991; Srinivasarao and Berry 1991; Magda et al. 1991).

A similar molecular theory has recently been applied to thermotropic small-molecule nematics composed of prolate molecules. Specifically, Archer and Larson (1995) and Kröger and Sellers (1995) have shown that in the limit of slow flows, where Brownian and excludedvolume terms nearly cancel, an approximate analytical expression for $\lambda$ may be derived in terms of the molecular aspect ratio, $a$, and the equilibrium values of the second and fourth moments of the orientation distribution function, $P_{2}=\left[3\left\langle\cos ^{2} \theta\right\rangle-1\right] / 2$ and $P_{4}=\left[35\left\langle\cos ^{4} \theta\right\rangle-30\left\langle\cos ^{2} \theta\right\rangle+3\right] / 8$. Here, $\theta$ is the angle between the orientation of the long axis of a molecule and the director, and $\langle\ldots\rangle$ denotes an average over the orientation distribution function. They derive an expression for $\lambda$ :

$\lambda=L(a) \frac{\left(5 P_{2}+16 P_{4}+14\right)}{35 P_{2}}$,

where $L(a)=\left(a^{2}-1\right) /\left(a^{2}+1\right)$. In Eq. (3), $P_{2}$ and $P_{4}$ can be considered as material parameters as long as the equilibrium form of the orientation distribution is not modified by the flow. This will be true for small-molecule nematics for all but extremely high shear rates (though it could be applied to polymeric nematics in the limit of very slow flows). When the Maier-Saupe nematic potential (Maier and Saupe 1959) is used to calculate the equilibrium values for $P_{2}$ and $P_{4}$, Eq. (3) predicts that near the clearing temperature, $T_{\mathrm{ni}}, \lambda$ increases with an increase in reduced temperature, $T_{\mathrm{r}}=T / T_{\mathrm{ni}}$. The effect of a finite aspect ratio, $a$, is to shift $\lambda$ to lower values than that predicted for an infinite aspect ratio. Estimates of aspect ratios typically range from 4 to $10 \mathrm{in}$ small-molecule liquid crystals, based on molecular bond length and angle data (Lide 1994). However, measured values of $\lambda$ for various liquid crystals (LCs), when plotted against $T_{\mathrm{r}}$, show that an infinite aspect ratio $(L(a)=1)$ often provides a better fit to the data (Archer and Larson 1995).

Equation (3) predicts that a small-molecule nematic is flow-aligning $(\lambda>1)$ close to the clearing point, but when the temperature is lowered, $\lambda$ decreases and the nematic becomes a tumbling one. The qualitative trend of decreasing $\lambda$ with decreasing temperature, as predicted by Eq. (3), has been established for several singlecomponent, small-molecule nematics. However, most single-component nematics have only a narrow range of temperatures over which they are nematic, and most of them crystallize or enter into the smectic-A phase before the temperature becomes low enough that a transition to tumbling might be expected according to Eq. (3).
An exceptional case is that of nematics which have a smectic-A phase between the nematic and the crystalline phase. These nematics are known to be especially prone to tumbling behavior, because of the presence in the nematic of pre-transitional smectic fluctuations (Bruinsma and Safinya 1991). These fluctuations can induce tumbling behavior even at reduced temperatures, $T_{\mathrm{r}}$, well above those for which tumbling is predicted by Eq. (3). The liquid crystal (LC) 8CB (4'-n-octyl-4-oxycyanobiphenyl), for example, has a phase transition to a smectic-A phase, and shows tumbling behavior at high $T_{\mathrm{r}}$.

The temperature range over which a nematic phase exists can be broadened considerably by mixing together two or more nematic-forming compounds. The use of mixtures to obtain a broad temperature range of nematic behavior is important in the LC display industry. Rey (1996) developed a generalization of Ericksen's transversely isotropic fluid model (TIF) for binary mixtures that allows one to predict the tumbling parameter of the mixture, $\lambda_{\mathrm{o}}^{\text {mix }}$, based on the tumbling parameters of the individual components, $\lambda^{\mathrm{n}}$ and $\lambda^{\mathrm{m}}$, and the relative volume fractions of the two components, $\Phi$ :

$\lambda_{\mathrm{o}}^{\operatorname{mix}}=\frac{\Phi R}{\Phi(R-1)+1} \lambda^{\mathrm{n}}+\frac{(1-\Phi)}{\Phi(R-1)+1} \lambda^{\mathrm{m}}$,

where $R$ is a concentration dependent ratio of the rotational viscosities of the two components in the mixture, $R(\Phi)=\gamma_{1}^{\mathrm{n}} / \gamma_{1}^{\mathrm{m}}$, and the subscript "o" indicates a slow flow regime, where the mixture remains uniaxial. The mixture becomes biaxial (i.e., having two directors) for stronger flows when the elastic restoring mechanism toward uniaxiality is weaker than the flow-induced biaxiality. The actual shear rate at which the slow flow regime ends depends on the degree of dynamic coupling of the two components, as discussed in an earlier paper by Rey (1995). If we assume that $R$, the ratio of the rotational viscosities of the two components in the mixture, remains constant with concentration, then Eq. (4) predicts that $\lambda_{\mathrm{o}}^{\mathrm{mix}}$ varies linearly with concentration in the case where $R=1$, and shifts toward the component with the more dominant $\gamma_{1}$ in the cases $R \gg 1$ or $R \ll 1$.

Our goal in this work is first to test the molecular theory, Eq. (3), for its temperature dependence of $\lambda$ in pure nematic LCs, then to expand this test to multicomponent mixtures. Also, we intend to test, at least qualitatively, whether the generalized two director Leslie-Ericksen equations derived by Rey are applicable to our multi-component results. In the end, we hope to discover whether the single director Leslie-Ericksen equations should be limited only to single-component nematics or if they may be successfully applied to all uniaxial nematics whether they be pure or mixtures. 
We would therefore like to measure $\lambda$ for a variety of pure nematics as well as nematic mixtures, and seek a simple method for doing so. Values of $\lambda$ can be extracted from the experimental values of Leslie viscosities reported in the literature simply by using Eq. (2). However, measuring these viscosities is a difficult task, even for a single-component nematic, and errors in the individual viscosities might propagate to give an inaccurate measure of $\lambda$.

A direct method of obtaining $\lambda$ is to shear an $\mathrm{LC}$ at high rates and determine the direction of orientation of the optic axis of the LC, using conoscopy for example. For a flow-aligning nematic, $\lambda$ can thereby be obtained by finding the flow-alignment angle at large strains, and using Eq. (1). For a tumbling nematic, there is no flowalignment angle, but $\lambda$ can be obtained by fitting the time-dependent rotation of the optic axis after start-up of shearing to predictions of the Leslie-Ericksen theory, with $\lambda$ as a fitting parameter. If the director is initially in the flow direction, then a qualitative determination of whether the nematic flow-aligns or tumbles can be made just by observing in which direction the optic axis rotates when shearing flow commences.

There are also several indirect methods of estimating $\lambda$, which have some advantages of convenience over the direct method. One method, proposed by Mather, Pearson, and Burghardt (MPB) (1995), involves the imposition of an oscillatory strain on a nematic with an initial director orientation perpendicular to the solid surfaces that drive the shearing flow (i.e., a homeotropic initial condition). This initial orientation is established by imposing a homeotropic boundary condition on the director at the solid surfaces. The amplitude of rotation of the director, compared to that of the shearing strain, can then be used to estimate $\lambda$. This method has an advantage over the direct method in that the homeotropic boundary condition is particularly easy to impose. Its disadvantage is that with homeotropic boundary conditions there is only a quantitative and no qualitative change in behavior when $\lambda$ passes through unity and the nematic changes from flow-aligning to tumbling. Thus experimental uncertainties in the measured value of $\lambda$ could lead one to miscategorize a flow-aligning nematic as a tumbling one, or vice versa.

Another indirect method is to look for textures that are specific to tumbling nematics in shearing flows. One such texture is a sequence of twist walls, which appear on start-up of fast shearing flow in a torsional geometry (Mather et al. 1997). The presence of these walls indicates that the nematic is of the tumbling type, and their absence identifies the nematic as a flow-aligner. In the case of a tumbling nematic $(\lambda<1)$, the rate of radial motion of these twist walls could also be used to extract a numerical estimate of $\lambda$; however, no numerical estimate of $\lambda$ is possible in the case $\lambda>1$. This method, again, works with homeotropic boundary conditions.
A final indirect method for estimating $\lambda$ is to measure the shear stress during start-up of shearing flow in a cone-and-plate or plate-and-plate rheometer. Gu et al. $(1993,1994)$ have shown that for a tumbling nematic in an initially homeotropic orientation, the shear stress oscillates after the start-up of fast shearing. The oscillation period $\gamma_{p}$, in strain units, is related to $\lambda$ by:

$\lambda_{\mathrm{p}}=\frac{2 \pi}{\sqrt{1-\lambda^{2}}}$.

For a flow-aligning nematic in such a flow, there are no oscillations, and no accurate estimate of $\lambda$ is possible by this method.

In this paper, both the direct and indirect methods are used to estimate $\lambda$ for various single-component and multi-component small-molecule LCs. In the following sections we describe the methods used in more detail, with the results for each method presented in the section that follows.

\section{Experimental methods}

Materials

We studied five small-molecule LCs and three commercially available mixtures used in LC displays. The pure compounds are a cyanophenylcyclohexane (PCH-5), and the 4 '- $n$-alkyl-4-cyanobiphenyls $(5 \mathrm{CB}, 6 \mathrm{CB}, 7 \mathrm{CB}$, and $8 \mathrm{CB})$. The commercial mixtures studied were E7, ZLI-3449-100, and ZLI-3308. The composition of E7 by weight is reported to be: $51 \% 5 \mathrm{CB}, 25 \% 7 \mathrm{CB}, 16 \% 8 \mathrm{OCB}$, and $8 \% 5 \mathrm{CT}$, where $8 \mathrm{OCB}$ is $4^{\prime}-n$-octyl-4-oxycyanobiphenyl and $5 \mathrm{CT}$ is $4^{\prime}-n$-pentyl-4-cyanoterphenyl. The compositions of the other two commercial mixtures are unknown to us. The materials in the $\mathrm{n}-\mathrm{CB}$ series and the mixture E7 were obtained from EM Industries and used as received. PCH-5, ZLI-3449-100, and ZLI-3308 were obtained from E. Merck and used as received. The phase transition temperatures reported for these compounds (Karat and Madhusudana 1976; Abdoh et al. 1982) are given in Table 1.

We have also prepared two different binary mixtures of smallmolecule LCs; one a mixture of $5 \mathrm{CB}$ and $8 \mathrm{CB}$, and the other a mixture of $7 \mathrm{CB}$ and $8 \mathrm{CB}$. The phase behavior of the $7 \mathrm{CB} / 8 \mathrm{CB}$ mixtures was determined by differential scanning calorimetry (DSC) and is shown in Fig. 1. The phase behavior of the $5 \mathrm{CB} /$ $8 \mathrm{CB}$ mixture was determined by hot stage polarizing microscopy

Table 1 Liquid crystal transition temperatures ( $K$ crystal, $A$ smetic, $N$ nematic, $I$ isotropic)

\begin{tabular}{lclc}
\hline Liquid crystal & K-A or K-N $\left[{ }^{\circ} \mathrm{C}\right]$ & A-N $\left[{ }^{\circ} \mathrm{C}\right]$ & N-I $\left[{ }^{\circ} \mathrm{C}\right]$ \\
\hline 5CB & 22.4 & - & 34.5 \\
6CB & 13.8 & - & 28.8 \\
7CB & 28.5 & - & 41.9 \\
8CB & 20.5 & 33.3 & 40.1 \\
PCH-5 & 30.0 & - & 55.0 \\
E7 & -30 & - & 58 \\
ZLI-3449-100 & -40 & - & 91 \\
ZLI-3308 & -30 & - & 128 \\
\hline
\end{tabular}




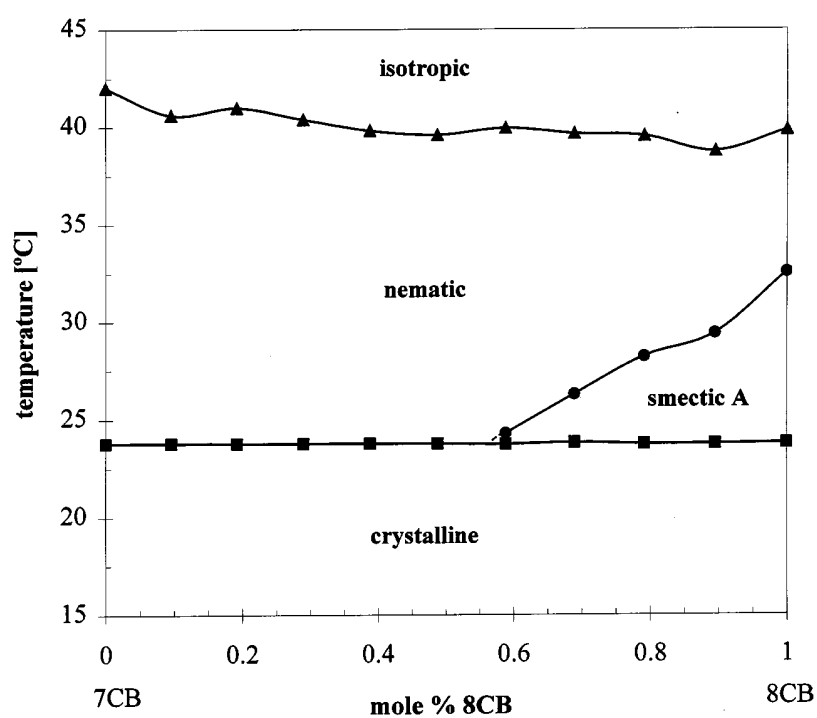

Fig. 1 Phase behaviour of $7 \mathrm{CB} / 8 \mathrm{CB}$ mixtures as determined by differential scanning calorimetry

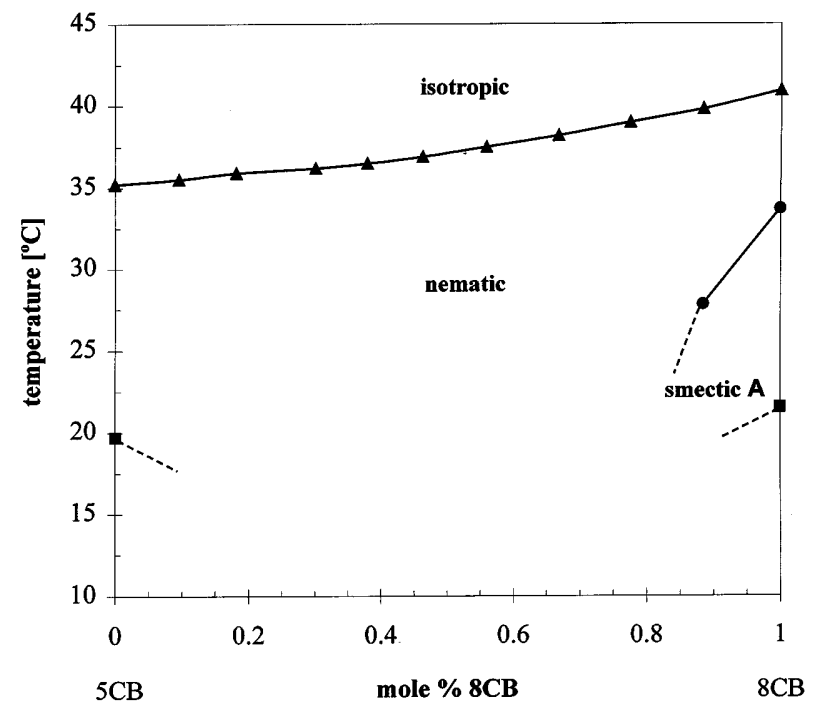

Fig. 2 Phase behavior of $5 \mathrm{CB} / 8 \mathrm{CB}$ mixtures as determined by hot stage polarizing microscopy

and is shown in Fig. 2. The crystalline transition in the $5 \mathrm{CB} / 8 \mathrm{CB}$ mixtures was below ambient temperature and hence undetermined.

\section{Surface alignment}

To make a meaningful connection between experimental observations and theoretical predictions, care must be taken to prescribe well-defined anchoring conditions on the director orientation. Planar alignment refers to the configuration where the LC molecules lie parallel to the surface, while for homeotropic alignment they are aligned perpendicular to the surface. Homeotropic boundary conditions are used in the MPB method for measuring $\lambda$, the twist wall experiments, and the rheological method. Planar alignment is used in the direct method.

Homeotropic alignment is obtained by treating the surfaces with a dilute solution of lecithin in chloroform. Excess lecithin is rinsed off with chloroform to make the surfaces transparent. The plates are then dried with a heating gun for approximately $2-3 \mathrm{~min}$. After loading the LC into the cell, it was found that uniform homeotropic alignment could be obtained in less than an hour.

Planar alignment is obtained by the usual method, using a rubbed polyimide film (Becker et al. 1986; Mosley et al. 1987). Uniform alignment is obtained in less than an hour. This treatment was found to give a director pretilt to the surfaces of around $2^{\circ}$.

\section{Conoscopy}

The method of conoscopy is used to observe the average angle of orientation of the LC across the gap of the shear cell. Only the details of the method relevant to us will be presented here. A more thorough treatment may be found in the books by Hartshorne and Stuart (1970), or Born and Wolf (1980).

The conoscopic image is generated by passing highly convergent monochromatic light through the sample placed between crossed polarizers. The interference figure is then viewed by focusing on the back focal plane of the objective lens using a Bertrand lens. A schematic of the experimental setup is shown in Fig. 3.

Since LCs are birefringent, rays of light entering the sample are doubly refracted, producing an extraordinary ray (E-ray) and an ordinary ray (O-ray). These rays travel at nearly the same angle through the sample but with different velocities, resulting in a relative phase shift, $\delta$, between them given by:

$\delta=\frac{d\left(n_{\mathrm{e}}^{*}\left(\theta_{\mathrm{E}}\right)-n_{\mathrm{o}}\right)}{\cos \phi}$,

where $d$ is the gap thickness, and $\phi$ is the angle of light propagation through the sample. The index of refraction that the E-ray sees, $n_{\mathrm{e}}^{*}\left(\theta_{\mathrm{E}}\right)$, depends on the angle that the vibration direction of the E-ray makes with respect to the director, $\theta_{\mathrm{E}}$, and is given by:

$n_{\mathrm{e}}^{*}\left(\theta_{\mathrm{E}}\right)=\frac{n_{\mathrm{e}} n_{\mathrm{o}}}{\sqrt{n_{\mathrm{o}}^{2} \cos ^{2} \theta_{\mathrm{E}}+n_{\mathrm{e}}^{2} \sin ^{2} \theta_{\mathrm{E}}}}$

where $n_{\mathrm{e}}$ and $n_{\mathrm{o}}$ are the principal indices of refraction of the LC. To predict the conoscopic image for a homeotropic or planar monodomain we need to express the retardance, $\delta$, strictly in terms of angles of light propagation through the sample, $\phi$. To do this, the orientation of the director with respect to the cone of light entering the sample should be considered so that angles with respect to the director, $\theta_{\mathrm{E}}$, can be written in terms of propagation

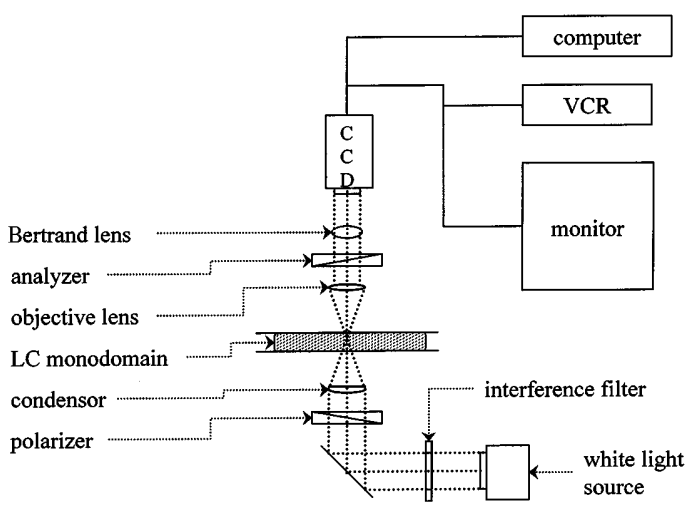

Fig. 3 Schematic of the experimental setup for conoscopy 
angles, $\phi$. As an example, in the homeotropic configuration, $\theta_{\mathrm{E}}=\phi+\pi / 2$, while in the planar configuration, $\theta_{\mathrm{E}}=\phi$.

The Bertrand lens brings rays of light travelling at the same angle into focus, causing the E- and O-rays to interfere, producing the conoscopic image. The intensity of light seen in the conoscopic image is then:

$I=I_{\mathrm{o}} \sin ^{2}\left(\pi \delta / \lambda_{\text {light }}\right) \sin ^{2}(2 \chi)$,

where $I_{\mathrm{o}}$ is the incident intensity, $\chi$ is the angle the incident light plane makes with respect to the polarizer direction, and $\lambda_{\text {light }}$ is the wavelength of light used.

The interference figure generated by a uniaxial crystal oriented in the homeotropic configuration consists of concentric dark rings called isochromes representing successive orders of birefringence and an extinction cross composed of what are called isogyres oriented parallel to the polarizer and analyzer directions. In the planar configuration, the isochromes, or bands of equal retardation, form hyperbolic fringes, which lie in four quadrants determined by the direction of orientation of the LC within the sample, with the polarizer and analyzer positioned at $45^{\circ}$ and $135^{\circ}$ with respect to the director. The center of symmetry of a planar conoscopic image may appear light or dark depending on the birefringence of the LC, and the thickness of the sample. Photographs of conoscopic images obtained for both homeotropic and planar configurations are shown in Fig. 4. The center of symmetry of each figure is centered within the field of view, indicating that the optic axis of the LC is exactly parallel or perpendicular to the axis of the cone of incident light for the two respective configurations, homeotropic and planar. For samples whose optic axis is inclined at an angle other than 0 or $90^{\circ}$ to that of the cone of light, the relationship between the symmetry point of the image and the angle of orientation in the sample is a bit more complicated and will be discussed next.

For samples with homeotropic alignment, the retardance is at a minimum for light travelling parallel to the optic axis of the LC. For this reason, the angular position of the cross in the homeotropic conoscopic image corresponds to light travelling parallel to the director. As the angle of orientation of the LC is inclined with respect to the optic axis of the microscope, the cross at the center of the image shifts from the center of the field of view accordingly. Hence, determining the instantaneous angle of the director orientation, in samples with small deviations from homeotropic alignment, is only a matter of determining the position of the cross in the conoscopic image. To determine the angular displacement of the director, knowing the displacement of the cross from the center of the field of view, a calibration factor is calculated between pixel displacement on the video screen and angular displacement of the director from homeotropic alignment within the sample. Knowing the indices of refraction of the LC
(Karat and Madhusudana 1976), the angular positions of the extinction rings seen in the conoscopic image of a quiescent sample are calculated. Then by measuring the number of pixels to the various rings, a calibration factor between angles of light through the sample and pixels seen on the video screen is determined. So for each image showing a displaced cross, simply counting the number of pixels between the cross and the center of a quiescent sample and multiplying by the calibration factor determines the angular rotation of the director away from homeotropic alignment.

In samples with planar alignment, the angular position of the symmetry point does not correspond directly to the overall tilt angle of the optic axis within the gap. A small tilt or deviation from uniform planar alignment leads to a much larger shift in the conoscopic image. This can be seen from Eq. (6), using the appropriate expression for $\theta_{\mathrm{E}}$ in $n_{\mathrm{e}}^{*}\left(\theta_{\mathrm{E}}\right)$. In Eq. (6), the birefringence is given by $n_{\mathrm{e}}^{*}\left(\theta_{\mathrm{E}}\right)-n_{\mathrm{o}}$, while the overall path length is given by $d / \cos \phi$. In the planar configuration, the birefringence decreases with $\phi$, while overall path length increases with $\phi$, leading to a complicated relationship between the angle of the symmetry point and the actual tilt angle within the sample. Scheffer and Nehring (1977) show that the tilt angle, $\alpha$, of the director measured from planar alignment may be determined knowing the indices of refraction of the $\mathrm{LC}$, and the angle of light propagation corresponding to the center of symmetry in the conoscopic image, $\phi_{x}$, using the rather complicated formula:

$$
\begin{aligned}
& \frac{\left(a^{2}-b^{2}\right)}{c^{2}} \sin \alpha \cos \alpha-\frac{a^{2} b^{2}}{c^{3}}\left(1-\frac{a^{2} b^{2}}{c^{2}} \sin ^{2} \phi_{x}\right)^{-1 / 2} \sin \phi_{x} \\
& +b\left(1-b^{2} \sin ^{2} \phi_{x}\right)^{-1 / 2} \sin \phi_{x}=0,
\end{aligned}
$$

where $a=1 / n_{\mathrm{e}}, b=1 / n_{\mathrm{o}}$, and $c^{2}=a^{2} \cos ^{2} \alpha+b^{2} \sin ^{2} \alpha$. As an example, for a LC with indices $n_{\mathrm{e}}=1.7, n_{\mathrm{o}}=1.5$, and a tilt angle, $\alpha=5^{\circ}$, the light propagation angle as seen on the conoscopic image is $\phi_{x}=16.3^{\circ}$. Angles of light propagation through the sample may be related to positions seen on the conoscopic image using the relation:

$$
\left(\frac{r}{R}\right) \mathrm{NA}=n_{\text {avg }} \sin \phi,
$$

where $r$ is a radial distance from the center of the field of view, $R$ is the total radius of the field of view, NA is the smaller of the numerical apertures of the objective lens and condensing lens, and $n_{\text {avg }}$ is the average refractive index of the sample, which may be approximated as $n_{\mathrm{avg}}=\frac{\left(n_{\mathrm{e}}+2 n_{\mathrm{o}}\right)}{3}$. Using this simple relation, we determine the angle of light propagation corresponding to various points on the image, which may be used in Eq. (9) to determine the tilt angle within the sample. As can be seen by the example above, it is important to use an objective lens that accepts a wide angle of light (high NA) to observe the symmetry point in planar samples
Fig. 4 Photographs of conoscopic images from a homeotropic and b planar liquid crystalline monodomains

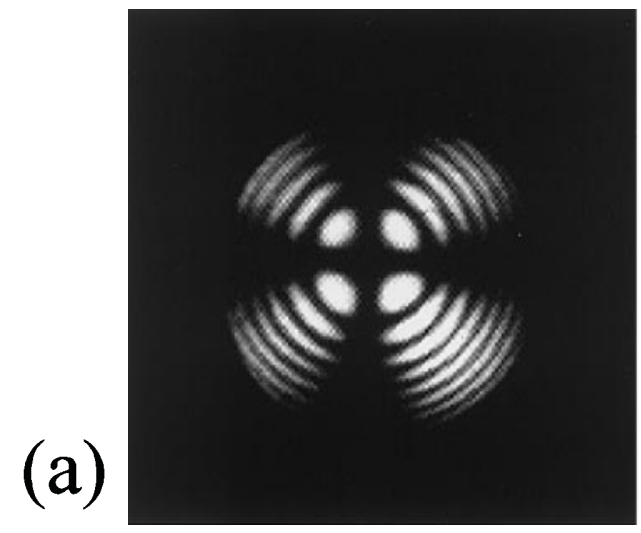

(b)

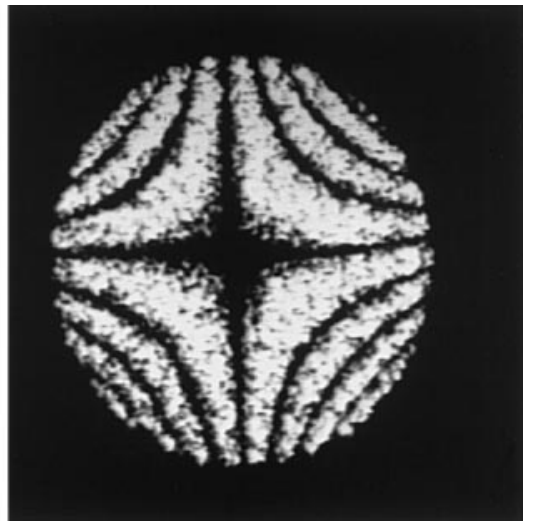


with substantial tilt angles. For the purposes of this work, it was sufficient to use a $40 \times$ objective with $\mathrm{NA}=0.5$.

\section{Methods of measuring $\lambda$}

MPB oscillatory method

Mather, Pearson, and Burghardt (Mather et al. 1995) solved the Leslie-Ericksen equations for the case of an applied oscillatory shear stress and found that certain material parameters may be obtained from the dynamic director response. In the case of homeotropic alignment, if the Leslie-Ericksen equations are linearized around $\psi=0$ (homeotropic alignment), one obtains the following expressions for the director angular momentum balance:

$\gamma_{1} \frac{\partial \psi}{\partial t}=K_{3} \frac{\partial^{2} \psi}{\partial y^{2}}+\left|\alpha_{2}\right| \frac{\partial v}{\partial y}$

and the constitutive relationship for shear stress, $\tau_{x y}$ :

$\tau_{x y}=\eta_{c} \frac{\partial v}{\partial y}-\left|\alpha_{2}\right| \frac{\partial \psi}{\partial t}$,

where $v$ is the velocity, $x$ is the flow direction, $y$ is the direction orthogonal to the plates, $K_{3}$ is the bend elastic constant, $\gamma_{1}=\alpha_{3}-\alpha_{2}$ is the rotational viscosity, $\eta_{c}=\left(-\alpha_{2}+\alpha_{4}+\alpha_{5}\right) / 2$ is a Miesowicz viscosity, and $\psi$ is the angle the director makes with respect to the $y$ direction. MPB use $\left|\alpha_{2}\right|$ in the analysis since they assume that $\alpha_{2}$ is negative, as is almost always the case for rod-like nematics. Applying an oscillatory shear stress, $\tau_{x y}=\tau_{0} \cos \omega t$, and neglecting fluid inertia, one solves Eqs. (11) and (12) for the amplitude ratio, $R^{*}$, as a function of frequency, and the LeslieEricksen material parameters. This amplitude ratio, $R^{*}=\psi_{0} / \gamma_{0}$, is defined as the ratio of the amplitude of the director response, $\psi_{0}$, to the amplitude of the applied strain, $\gamma_{0}$.

In the high-frequency regime, MPB find that the amplitude ratio, $R^{*}$, is independent of frequency and given by:

$\lim _{\omega \rightarrow \infty} R^{*}=\frac{\left|\alpha_{2}\right|}{\gamma_{1}}$.

By a simple algebraic manipulation using $\gamma_{1}=\alpha_{3}-\alpha_{2}$, the tumbling parameter, $\lambda$, may be determined from the high-frequency amplitude ratio as:

$\lambda=2 R_{\infty}^{*}-1$.

To measure the amplitude ratio a torsional flow cell is used, consisting of two parallel disks mounted in the optical path of a microscope set up for conoscopy (Mather 1994). The applied strain, imposed by rotating one disk with respect to the other, is defined as $\gamma=r \Omega / h$, where $r$ is the observation radius, $\Omega$ is the angular displacement of the plate, and $h$ is the sample thickness. Experiments were conducted away from the center of the cell to minimize the effects of curved streamlines. Strain amplitudes in our experiments range from $3-10 \%$ depending on the frequency, $\omega$. The amplitude of the oscillatory director response, $\psi_{0}$, is determined using the method of conoscopy. The conoscopic images are recorded with a CCD camera and viewed on a video monitor. A frame grabber saves the images throughout the experiment for later viewing. Each image saved throughout the cycle shows a conoscopic image whose center is displaced from that of a quiescent sample, indicating that the director is angularly displaced at that instant. For each image, simply measuring the displacement, in pixels, of the center of the image from that of the quiescent image and using the calibration factor discussed previously, determines the average director angle within the gap corresponding to that image. By analyzing images throughout the cycle, the oscillatory strain, $\gamma(t)$, and the resulting oscillatory director response, $\psi(t)$ may be plotted. We then determine both the strain amplitude, $\gamma_{0}$, and the director response amplitude, $\psi_{0}$, from these sinusoidal curves. The tumbling parameter is then determined from the amplitude ratio in the high-frequency limit using Eq. (14).

As mentioned in the Introduction, the MPB method, when used with homeotropic boundary conditions, has the disadvantage that there is no qualitative change in behavior when $\lambda$ passes through unity, that is, when the nematic changes from a tumbling to a flow-aligning system. For samples with planar alignment under oscillatory shear, the relative phase of the applied strain and the resulting director oscillation provide a qualitative distinction between tumblers and flow-aligners. A tumbling nematic in the high-frequency limit will oscillate in phase with the applied strain, while a flow-aligning nematic will oscillate $180^{\circ}$ out of phase with the applied strain. Unfortunately, the planar configuration is much more difficult to attain in this geometry, so all the experimental results reported for this method were obtained with homeotropic anchoring. Thus, considering that most small-molecule LCs have $\lambda$ values very near 1 , if one relies on this technique alone imprecision in the measurement might lead one to misclassify a flow-aligning nematic as a tumbling one, or vice versa. We therefore develop three other methods, two qualitative and one quantitative, for distinguishing between tumbling and flow-aligning nematics and to check for consistency among the methods.

\section{Twist wall experiment}

Textural observations characteristic of tumbling and flow-aligning behavior may also be used to distinguish between the two classes of LCs. One such textural observation is the appearance of what are called twist walls, in the torsional shear flow of an LC monodomain initially aligned in the homeotropic configuration. These twist walls were first observed and characterized in a paper by Mather et al. (1997). They use the term "twist wall" coined by Carlsson and Skarp (1986) to describe a region across which the director undergoes a jump in orientation due to a tumbling instability.

To observe these twist walls, the torsional shearing cell consisting of two parallel plates, described in the previous section, is used. The shearing is started at a high rate, with the LC initially aligned uniformly in the homeotropic configuration. Observing the flow cell between crossed polarizers, with the polarizer in the flow direction and the analyzer in the vorticity direction, twist walls appear as a series of birefringent lines, which propagate inward from the edge of the cell. Eventually the twist walls give way to disclination lines, which fill the cell. For a flow-aligning nematic, on the other hand, the director angle decreases toward the flowalignment angle with increasing shear rate, and no twist walls will appear.

The proposed mechanism (Mather et al. 1997) for twist wall formation is as follows. As the flow is started from rest, the director of a tumbling nematic will continuously rotate in the shearing plane, with the rate of rotation dependent on the current orientation angle of the director and the shear rate at that radial position. The director rotates fastest near the edge of the shear cell, where the shear rate is the greatest. The director is especially susceptible to rotation out of the shearing plane, by a mechanism analogous to a mechanical buckling instability each time it passes through an angle parallel to the flow direction. The shearing plane here is defined as the plane containing both the flow direction and the velocity-gradient direction. Careful conoscopic studies by Mather et al. (1997) on the director orientation in the vicinity of an approaching twist wall reveal a gradual rotation of the director out of the shear plane towards an orientation in the vorticity direction at the twist wall position, with the orientation presumably undergoing a gradual twist back into the plane of shear as the wall moves past. As the torsional shearing proceeds, these twist walls form at the edge of the cell and propagate radially inward, marking 
regions within the cell in which the director is rotating through an angle parallel to the flow direction.

Thus, in a sudden onset of torsional shearing, the presence or absence of these twist walls provides a qualitative means of determining whether or not the nematic is of the tumbling type. The rate of radial propagation of these twist walls might also be used to determine the tumbling parameter quantitatively, although that hasn't been exploited in this work. In this experiment it is necessary to start at a high shear rate to generate twist walls before disclinations form by some other mechanism.

\section{Direct method}

By observing the conoscopic image of a sample with an initial planar alignment in shear flow, it is possible to distinguish between tumbling and flow-aligning behavior by simply observing in which direction the conoscopic image shifts upon shear start-up.

Glass slides treated with a rubbed polyimide film are used to induce a planar alignment. Cover slips with a thickness of 170 microns are used as spacers at each end of the two slides. A small amount of the LC is placed in the center of the slide and the top slide is brought to rest on the spacers. A schematic of the shearing cell is shown in Fig. 5. This shearing cell, consisting of an LC sandwiched between two slides, is placed in a Teflon frame which holds the slides firmly in place against the cover-slip spacers, while allowing one slide to be pushed over the other. The shearing cell is then placed in the optical path of a microscope set up for conoscopy. After allowing an hour for the LC to align, an image of a planar sample with a small pre-tilt $\left(2-3^{\circ}\right)$ appears. Now pushing the top slide over the bottom slide, and noting the direction of the shift in the conoscopic image, we can determine whether the director in the bulk rotates up toward a flow- alignment angle, or in the opposite direction rotating with vorticity. If the flow-alignment angle is small enough that the symmetry point in the conoscopic image remains in the field of view while shearing, an estimate of the flow-alignment angle and of $\lambda$ may be obtained by measuring the displacement of the image. The essential point of this experiment, however, is not to measure $\lambda$ quantitatively, but only to distinguish between tumbling and flow-aligning behavior. Since the plates are sheared by hand the flow-alignment angles obtained by this method are only estimates. Also, the shear cell is not temperature controlled so the results shown are for room-temperature nematics only.

\section{Rheological method}

The tumbling parameter, $\lambda$, for tumbling nematics may also be determined by a simple rheological method first demonstrated by Yang and Shine (1993) and soon after by $\mathrm{Gu}$ et al. (1993). A homeotropic monodomain is formed in a cone-plate rheometer. Upon start-up of shear, the resulting shear stress or apparent viscosity is measured. For tumbling nematics the shear stress oscillates with a strain period characteristic of the tumbling parameter of the material. Flow-aligning nematics, on the other

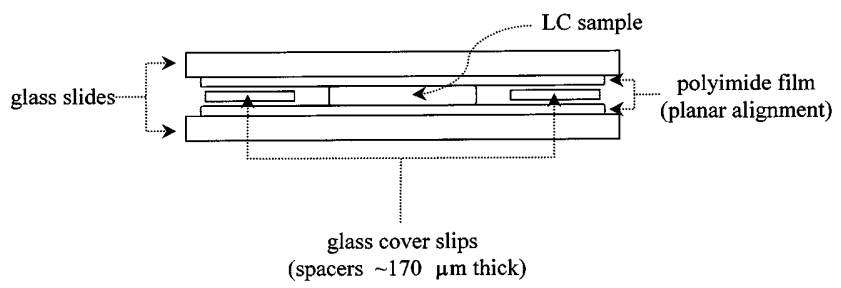

Fig. 5 Schematic showing the shear cell for direct measurements of tumbling versus flow-alignment hand, show only an initial overshoot in the shear stress, followed by a constant viscosity.

The different responses may be understood by considering the predictions of Ericksen's TIF model (Ericksen 1960). We use a twodimensional coordinate system in which $x$ is the flow direction and $y$ is the velocity-gradient direction. Then, neglecting elasticity and assuming a constant shear rate, $\dot{\gamma}=\partial v / \partial y$, a torque balance for the director in this geometry gives:

$\frac{\partial \theta}{\partial \gamma}=\frac{\left(\alpha_{3} \sin ^{2} \theta-\alpha_{2} \cos ^{2} \theta\right)}{\left(\alpha_{3}-\alpha_{2}\right)}$,

where $\theta$ is the angle the director makes with respect to the $y$ direction, $\gamma$ is the dimensionless time or strain, and the $\alpha_{i}$ 's are the Leslie coefficients. Integrating (15) with the initial condition that $\theta=0$ at $\gamma=0$, and making the substitution, $\alpha_{2} / \alpha_{3}=(\lambda+1) /$ $(\lambda-1)$, gives for the two cases, $\alpha_{3}<0$ (flow-aligning) and $\alpha_{3}>0$ (tumbling):

$\gamma=\frac{1}{\sqrt{\lambda^{2}-1}} \log \left[\frac{1+\sqrt{\frac{\lambda-1}{\lambda+1} \tan \theta}}{1-\sqrt{\frac{\lambda-1}{\lambda+1} \tan \theta}}\right], \quad$ (flow-aligning)

and

$\gamma=\frac{2}{\sqrt{1-\lambda^{2}}} \operatorname{atan}\left(\sqrt{\frac{1-\lambda}{1+\lambda}} \tan \theta\right), \quad$ (tumbling)

For the tumbling case, the stress oscillates with a strain period, $\gamma_{p}$, of

$\gamma_{\mathrm{p}}=\frac{2 \pi}{\sqrt{1-\lambda^{2}}}$

To determine the orientations that correspond to the various peaks and valleys in the shear stress response, we start with the equation for the shear stress, $\tau_{x y}$, for an initial director alignment perpendicular to the flow direction and parallel to the velocity gradient,

$$
\begin{aligned}
\tau_{x y}=\dot{\gamma} & \left\{\alpha_{1} \cos ^{2} \theta \sin ^{2} \theta+\eta_{\mathrm{c}}+\left(\alpha_{2}+\alpha_{3}\right) \sin ^{2} \theta\right. \\
& \left.-\frac{\left(\alpha_{2} \cos ^{2} \theta-\alpha_{3} \sin ^{2} \theta\right)^{2}}{\left(\alpha_{3}-\alpha_{2}\right)}\right\},
\end{aligned}
$$

where $\eta_{\mathrm{c}}=\left(-\alpha_{2}+\alpha_{4}+\alpha_{5}\right) / 2$ is the Miesowicz viscosity when $\theta=0$. Now, taking the derivative of $\tau_{x y}$ with respect to $\theta$, it has been shown (Viola and Baird 1986; Yang and Shine 1993) that for a flow-aligning nematic, $\alpha_{3}<0$, the shear stress has a single maximum at $\theta=\pi / 4$; while for a tumbling nematic, $\alpha_{3}>0$, a series of maxima in the shear stress occur at $\theta=m \pi / 4(m=$ odd $)$, and a series of minima appear at $\theta=n \pi / 2(n=0,1,2,3, \ldots)$ subject to the condition that $\alpha_{1}>\left(\alpha_{2}+\alpha_{3}\right)^{2} /\left(\alpha_{3}-\alpha_{2}\right)$. Knowing that the stress is a maximum at $\theta=\pi / 4$, one can also derive the strain of the first stress overshoot, $\gamma_{0}$, for flow-aligning and tumbling nematics, from Eq. (16) and (17):

$\gamma_{0}=\frac{\mathrm{a} \cosh (\lambda)}{\left(\lambda^{2}-1\right)}, \quad$ (flow-aligning)

and

$\gamma_{0}=\frac{2}{\sqrt{1-\lambda^{2}}} \operatorname{atan}\left(\sqrt{\frac{1-\lambda}{1+\lambda}}\right)$. (tumbling)

In theory, $\gamma_{0}$ could be used to distinguish between tumbling and flow-aligning nematics since Eqs. (20) and (21) predict that $\gamma_{0}<1$ for flow-aligning nematics and $\gamma_{0}>1$ for tumbling nematics. 
Unfortunately, this is an unreliable method of distinguishing between the two behaviors since $\lambda$ typically varies between 0.8 and 1.2 , for the small-molecule nematics studied here, corresponding to $\gamma_{0}$ varying between 1.07 and 0.94 respectively, which is a variation less than accuracy with which we can determine the stress maximum.

\section{Results and discussion}

MPB method results

The MPB method was used to measure quantitatively the tumbling parameter of some pure small-molecule LCs and some mixtures of small-molecule LCs. All the experiments using this method were conducted with the director initially oriented in the homeotropic configuration.

The first measurements were conducted on $7 \mathrm{CB}$, a system where we could expect the Leslie-Ericksen theory that underlies the interpretation of this method to be valid. We used this method to determine the temperature dependence of the tumbling parameter over a small range of reduced temperatures, $T_{\mathrm{r}}=T / T_{\mathrm{ni}}$, near the clearing point. The tumbling parameter was found to increase with reduced temperature from $\lambda=0.98$ at $T_{\mathrm{r}}=0.97$ to $\lambda=1.07$ at $T_{\mathrm{r}}=0.99$. The temperature dependence is qualitatively similar to experimental results reported for other small-molecule LCs and obtained by other methods, as well as the behavior predicted by the molecular theory of Archer and Larson, and Kröger and Sellers. The results measured for $7 \mathrm{CB}$ using the MPB method are, however, slightly lower than those reported for other small-molecule LCs (Gähwiller 1972; Kneppe et al. 1981, 1982, 1981; Graf et al. 1992). Figure 6 shows the temperature dependence found for $7 \mathrm{CB}$, along with the temperature dependence reported for other small-molecule LCs as determined by other methods (Gähwiller 1972; Kneppe et al. 1981, 1982, 1981; Graf et al. 1992). The lines in Fig. 6 correspond to molecular theory, Eq. (3), using Maier-Saupe (Maier and Saupe 1959) predictions for the equilibrium values of $P_{2}$ and $P_{4}$, for both an infinite aspect ratio and a more realistic aspect ratio of $a=5$.

In an attempt to understand how the tumbling parameter behaves for mixtures of small-molecule LCs, the MPB method was used to measure $\lambda$ for two binary mixtures, $5 \mathrm{CB}$ with $8 \mathrm{CB}$, and $7 \mathrm{CB}$ with $8 \mathrm{CB}$, at various concentrations. The first binary mixture studied was $7 \mathrm{CB} / 8 \mathrm{CB}$. The phase behavior of this mixture, determined by DSC scans, is shown in Fig. 1. As reported above, $7 \mathrm{CB}$ has a tumbling parameter, $\lambda$, near unity throughout its nematic range, while $8 \mathrm{CB}$ has a much lower tumbling parameter due to the pre-transitional effects of a nearby smectic-A phase transition at $33.3{ }^{\circ} \mathrm{C}$. $\lambda$ values were determined at a single temperature, $33.6{ }^{\circ} \mathrm{C}$, for a few mixtures of the tumbling nematic,

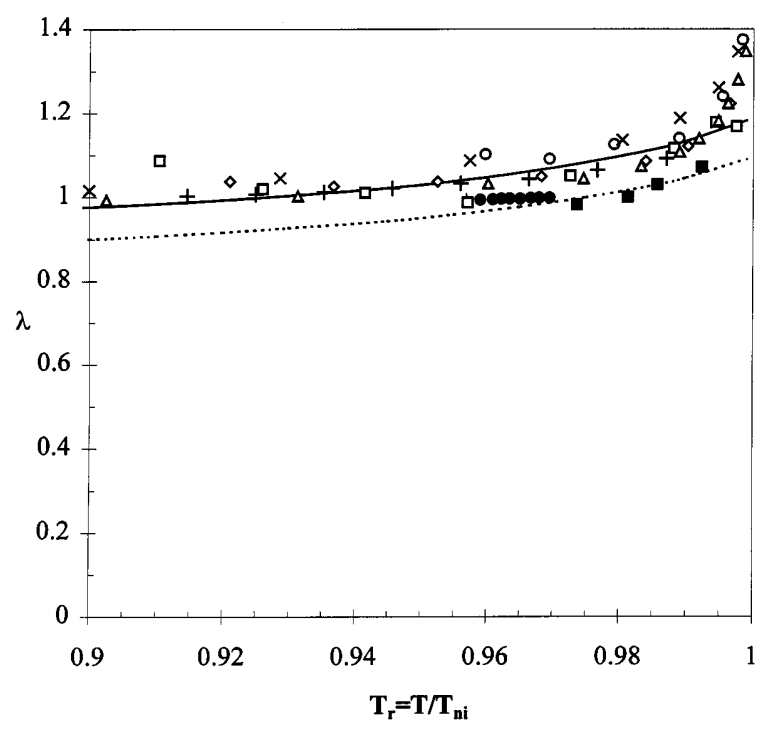

Fig. 6 Measured $\lambda$ versus $T_{\mathrm{r}}$ for $7 \mathrm{CB}$, as obtained by the MatherPearson-Burghardt (MPB) method (ם), and the rheological method (O). Results for other small-molecule liquid crystals: 5CB (O), EM $(\square)$ MBBA $(\diamond), \mathrm{N} 4(\times), \operatorname{HBAB}(+)$, and N5 $(\Delta)$, obtained from measurements of the individual Leslie viscosities reported by (Gähwiller 1972; Kneppe et al. 1981, 1982, 1981; Graf et al. 1992) are shown for comparison. Also, the prediction of the molecular theory of Archer and Larson (1995) is shown for both an infinite aspect ratio (-) and for an aspect ratio of $a=5(---)$

$8 \mathrm{CB}$, with the flow-aligning nematic, 7CB. Values of $\lambda$ were found to vary linearly with the $8 \mathrm{CB}$ volume fraction, as shown in Fig. 7. The error bars are from the standard deviation of the high-frequency data.

The phase behavior of the other binary mixture, $5 \mathrm{CB}$ with $8 \mathrm{CB}$, was determined by hot stage polarizing microscopy, and is shown in Fig. 2. As with the $7 \mathrm{CB} /$ $8 \mathrm{CB}$ mixtures, the tumbling parameter was determined over a range of concentrations at a single temperature, $33.6^{\circ} \mathrm{C}$, at which the nematic ranges of the two pure components overlap. Similar to the $7 \mathrm{CB} / 8 \mathrm{CB}$ mixtures, $\lambda$ values in the $5 \mathrm{CB} / 8 \mathrm{CB}$ mixtures were found to vary linearly with the volume fraction of $8 \mathrm{CB}$, as shown in Fig. 8. It would be interesting to know whether this linear relationship holds for other binary or multicomponent mixtures, including mixtures of dissimilar molecules where the nematic ranges do not overlap, and if these results are consistent with those obtained by the other quantitative method for measuring $\lambda$, namely the rheological method.

In hopes of testing the molecular theory for $\lambda$, Eq. (3), over a wider range of temperatures than was accessible with single-component $\mathrm{LCs}$ such as $7 \mathrm{CB}$, we turned to commercial mixtures. The commercial mixtures studied were E7, ZLI-3449-100, and ZLI-3308. $\lambda$ was measured for each of these mixtures at the same four temperatures ranging from room temperature up to $55^{\circ} \mathrm{C}$. None of these results from the MPB method, 


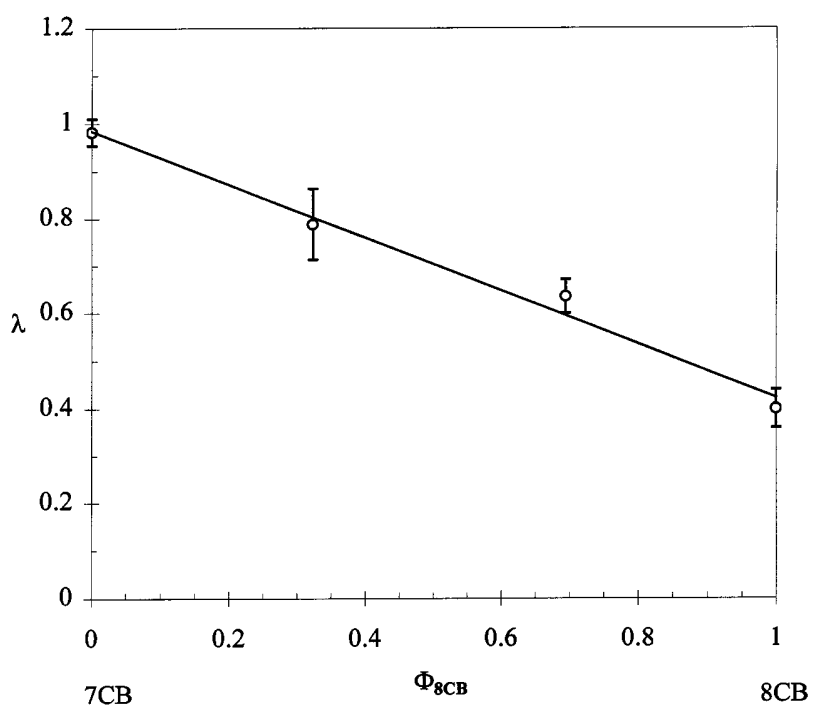

Fig. $7 \lambda$ versus volume fraction for $7 \mathrm{CB} / 8 \mathrm{CB}$ mixtures at $33.6^{\circ} \mathrm{C}$, as determined by the MPB method. Error bars are determined from the standard deviation of $R^{*}$ in the high frequency asymptote

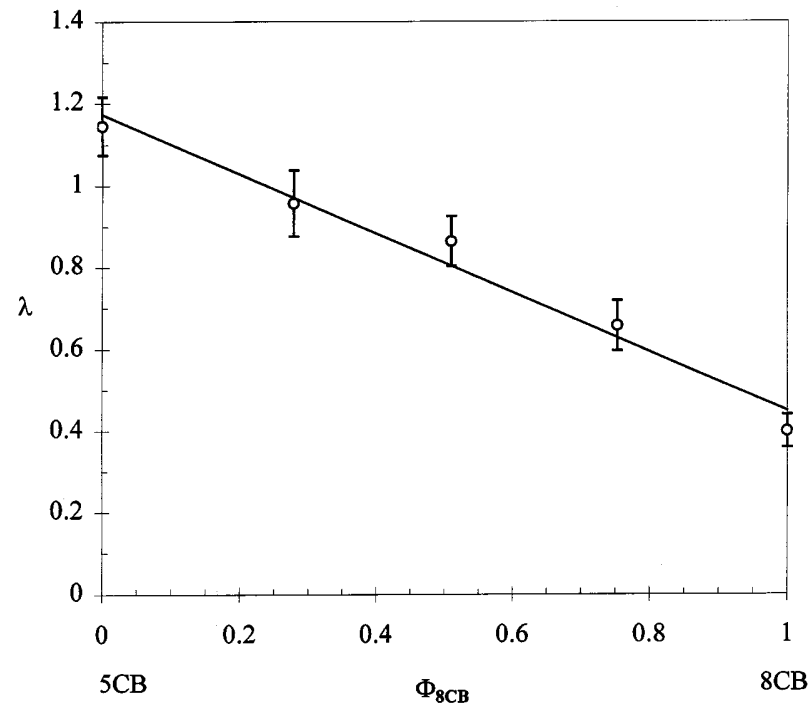

Fig. $8 \lambda$ versus volume fraction for $5 \mathrm{CB} / 8 \mathrm{CB}$ mixtures at $33.6^{\circ} \mathrm{C}$, as determined by the MPB method. Error bars are determined from the standard deviation of $R^{*}$ in the high frequency asymptote. Line shown represents the best straight line fit

when plotted against reduced temperature, Fig. 9, may be fit quantitatively by the molecular theory of Archer and Larson (1995). E7 shows a nearly constant $\lambda$ value, ranging from 0.83 to 0.86 , without the characteristic increase near the clearing point as the molecular theory predicts. $\lambda$ for ZLI-3449-100 increases slightly with increasing temperature, similar to that predicted by the molecular theory; however, the values range from 0.66 to 0.74 , well below that predicted for even the low aspect

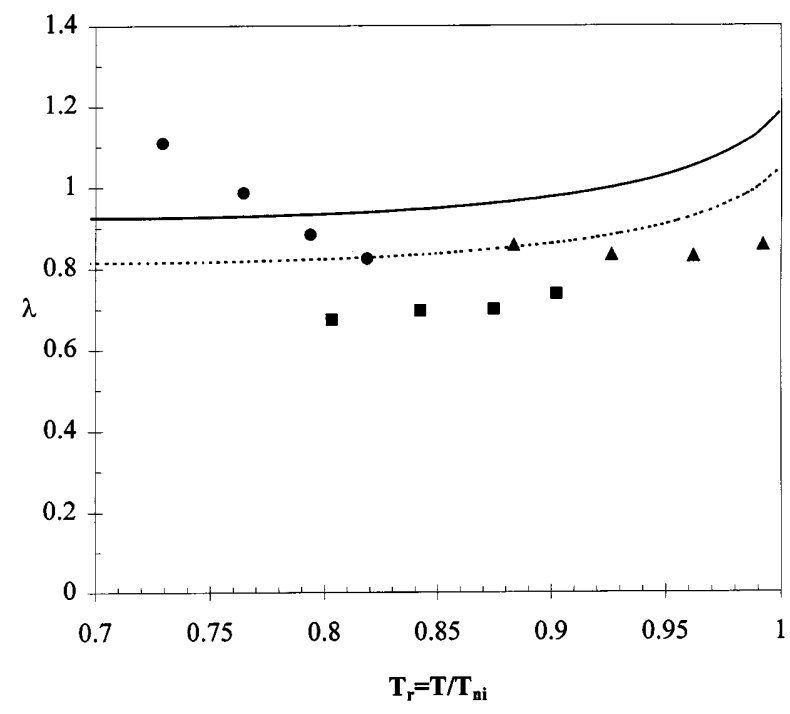

Fig. 9 Measured $\lambda$ versus $T_{\mathrm{r}}$ for the commercial mixtures E7 (@), ZLI3449-100 (ם), and ZLI-3308 ( $\mathbf{\Delta})$ by the MPB method. Also, the prediction of the molecular theory of Archer and Larson (1995) is shown for both an infinite aspect ratio (-) and for an aspect ratio of $a=4(---)$

ratio of $a=4$. ZLI-3308 behaves unlike the molecular theory and any other small-molecule LC in that the tumbling parameter decreases sharply form $\lambda=1.11$ to $\lambda=0.83$ with an increase in temperature from room temperature up to $55.4^{\circ} \mathrm{C}$.

The results for the single-component $\mathrm{LC}, 7 \mathrm{CB}$, are in agreement with the temperature dependence predicted by the molecular theory, Eq. (3), as well as the temperature dependence exhibited by other single-component LC systems found in the literature. The results for the multi-component mixtures, E7, ZLI-3449-100, and ZLI-3308, however, are not even qualitatively similar to that of the molecular theory. This result alone suggests either a breakdown in the single director LeslieEricksen theory, which the MPB method is based on, or a failure in the molecular theory to describe the dynamics of multi-component systems when MaierSaupe predictions for the equilibrium values of $P_{2}$ and $P_{4}$ (originally derived for single-component systems) are used. The results for the binary mixtures, $5 \mathrm{CB} / 8 \mathrm{CB}$ and $7 \mathrm{CB} / 8 \mathrm{CB}$, show a nearly linear concentration dependence of $\lambda$.

\section{Twist wall observations}

Twist wall experiments were conducted on a couple of the materials in this study to determine whether the predictions of the MPB method are consistent with the textural observations.

The first material studied, $8 \mathrm{CB}$, known to be a tumbler due to its nearby smectic-A phase, was aligned 
homeotropically in the shear at $36^{\circ} \mathrm{C}$. Upon start-up of shear at a high shear rate $\left(\dot{\gamma}>1 \mathrm{~s}^{-1}\right)$, several twist walls were seen to propagate inward from the edge of the cell. After continued shearing, the cell becomes filled with disclination lines. These observations are similar to those made by Mather (1994), and confirm that $8 \mathrm{CB}$ is a tumbling nematic at $36^{\circ} \mathrm{C}$.

The other material studied by this method was the commercial mixture, E7. According to the MPB method, $\lambda$ for E7 is less than unity; hence E7 should tumble at temperatures ranging from room temperature up to $55^{\circ} \mathrm{C}$. Since most studies on LCs have reported tumbling only at temperatures near a transition to a smecticA phase, these results from the MPB method are surprising. At room temperature, and at shear rates identical to those used in the 8CB twist wall experiments $\left(\dot{\gamma}>1 \mathrm{~s}^{-1}\right)$, no twist walls were found after continued shearing. This observation suggests that E7 is actually a flow-aligner at room temperature rather than a tumbler as the MPB method indicates.

Again, the single-component result is consistent with that of the MPB method, while the result for the multicomponent mixture disagrees with that predicted by the MPB method.

\section{Direct method results}

Since the MPB method and the twist wall observations give contradictory indications for E7, the direct method was used to determine definitively whether the LCs studied by the MPB method are tumblers or flowaligners. The experiments were conducted at room temperature on the following LCs: $5 \mathrm{CB}, 6 \mathrm{CB}, 7 \mathrm{CB}$, PCH-5, E7, ZLI-3449-100, and ZLI-3308. At room temperature, the LCs $7 \mathrm{CB}$ and $\mathrm{PCH}-5$ are both supercooled nematics.

The samples were sheared by hand, with the top plate displaced around $4 \mathrm{~mm}$ in less than a second. With spacers of 170 -micron thickness, the shear rate, $\dot{\gamma}$, is estimated to be of the order of $20 \mathrm{~s}^{-1}$, and the shear strain around 25. The Ericksen number, $\mathrm{Er}=(\eta / K) \dot{\gamma} h^{2}$, is therefore estimated to be of the order of $10^{2}-10^{3}$, based on typical values of the Leslie coefficients and Frank constants. At such high Ericksen numbers, hydrodynamic effects are expected to dominate over elastic effects, and one can therefore obtain estimates of the tumbling parameter, $\lambda$, from the observed flowalignment angles based on Eq. (1).

The results of the direct method are summarized in Table 2. The flow-alignment angles are estimated from the displacement of the conoscopic image during shearing. For those systems with high flow-alignment angles in which the image displaces out of the field of view, only lower bounds for the flow-alignment angle, $\theta_{\mathrm{fa}}$ and $\lambda$ are obtained. For those LCs for which the conoscopic image remains within the field of view, upper and lower bounds are obtained; these correspond to limits estimated from repeated experiments. The only system found to tumble in these experiments was $7 \mathrm{CB}$. For $7 \mathrm{CB}$, shearing causes the image to shift in the direction of a tumbler, after which the image disappears as the cell fills with disclinations. The formation of disclinations on shearing is typical of tumbling nematics. Upon shearing of the $\mathrm{PCH}-5$ sample, the image displaces in the direction of a flow-aligner but the metastable nematic quickly crystallizes; hence, no flow-alignment angle could be determined.

Here, the single-component results for $5 \mathrm{CB}$ and $7 \mathrm{CB}$ agree in their prediction for flow-aligning and tumbling with those obtained by the MPB method, while the results for the mixtures, E7 and ZLI-3449-100, disagree with those obtained by the MPB method.

\section{Rheological method results}

The rheological method was used on the following materials: 5CB, 7CB, 8CB, E7, PCH-5, ZLI-3308, and ZLI-3449-100. The method was also used on two binary mixtures. One is a mixture of a flow-aligning nematic, $5 \mathrm{CB}$, with a tumbling nematic, $8 \mathrm{CB}$, and the other a mixture of two tumbling nematics, $7 \mathrm{CB}$ and $8 \mathrm{CB}$, with very different tumbling parameters. The entire nematic range was studied for each material except for the commercial mixtures, E7, ZLI-3449-100 and ZLI-3308, where the lower transition to the smectic or crystalline phase was beyond the temperature range of our rheometer. Also, the clearing temperature of ZLI-3308, $T_{\mathrm{ni}}=128^{\circ} \mathrm{C}$, was too high to be reached by our rheometer. The experiments were carried out in the cone-andplate geometry of a Rheometrics ARES rheometer, with a cone angle of 0.04 radians and a diameter of $50 \mathrm{~mm}$.

The loading procedure for this method was identical for all the materials studied here. The cone and plate fixtures were initially treated with a dilute solution of lecithin in chloroform and smeared to cover the entire surface of the fixtures. The fixtures were then allowed to

Table 2 Direct method results ${ }^{\mathrm{a}}$

\begin{tabular}{llll}
\hline Liquid crystal & Result & $\theta_{\text {fa }}$ & $\lambda$ \\
\hline 5CB & Flow-aligns & $8^{\circ}<\theta_{\text {fa }}$ & $1.04<\lambda$ \\
6CB & Flow-aligns & $9^{\circ}<\theta_{\text {fa }}$ & $1.05<\lambda$ \\
7CB & Tumbles & - & $\lambda<1$ \\
PCH-5 & Flow-aligns & $\mathrm{b}$ & $1<\lambda$ \\
E7 & Flow-aligns & $4^{\circ}<\theta_{\text {fa }}<7^{\circ}$ & $1.01<\lambda<1.03$ \\
ZLI-3449-100 & Flow-aligns & $6^{\circ}<\theta_{\text {fa }}<8^{\circ}$ & $1.02<\lambda<1.04$ \\
ZLI-3308 & Flow-aligns & $9^{\circ}<\theta_{\text {fa }}$ & $1.05<\lambda$ \\
\hline
\end{tabular}

\footnotetext{
${ }^{a}$ Results shown are obtained at room temperature

${ }^{\mathrm{b}} \theta_{\mathrm{fa}}$ unobtainable since super-cooled nematic recrystallized upon shearing
} 
sit for a period of 30 min to allow the chloroform to evaporate, leaving a rather uneven film of lecithin on the surfaces. The excess lecithin was removed by lightly rubbing the fixtures with a lint-free cloth, leaving the fixtures with a thin film of lecithin. The fixtures were then mounted on the rheometer, the LC sample was added, and the gap set. The sample was then left for approximately an hour to allow a homeotropic monodomain to form between the cone and plate.

To ensure that we could use this method to distinguish between tumbling and flow-aligning nematics, the first two materials studied were $5 \mathrm{CB}$ and $8 \mathrm{CB}$. This also allowed us to verify our procedure by obtaining quantitative comparisons with the earlier work by $\mathrm{Gu}$ and Jamieson (1994).

$5 \mathrm{CB}$ was tested at 0.5 degree increments over temperatures ranging from $23.1^{\circ} \mathrm{C}$ up to $36.4^{\circ} \mathrm{C}$. $5 \mathrm{CB}$ was found to flow-align throughout its entire nematic range with typical flow-aligning responses, shown in Fig. 10. Similar to the results obtained by $\mathrm{Gu}$ and Jamieson (1994), the height of the initial stress overshoot is a maximum at the lowest temperature and decreases steadily with increasing temperature until the nematicisotropic transition is reached. Since this material is flow-aligning and the strain accuracy of our rheometer is insufficient to accurately determine the position of the first stress overshoot, no estimate of the tumbling parameter was obtained from these curves.

To test the effectiveness of this method as a means of determining $\lambda$ for a tumbling nematic, $8 \mathrm{CB}$ was tested at 0.5 degree increments throughout most of its nematic range from $34.2{ }^{\circ} \mathrm{C}$ up to $39.3{ }^{\circ} \mathrm{C}$. Typical tumbling responses of $8 \mathrm{CB}$ are shown in Fig. 11. These curves are similar to those reported by Gu and Jamieson (1994) and

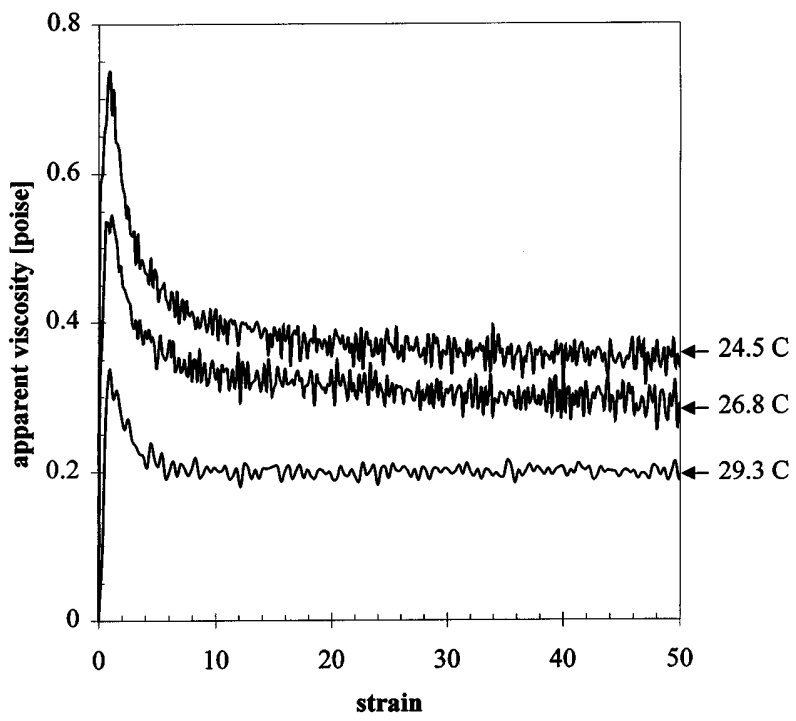

Fig. 10 Apparent viscosity versus strain for $5 \mathrm{CB}$ on shear start-up at $16 \mathrm{~s}^{-1}$ for three temperatures: $24.5,26.8$, and $29.3^{\circ} \mathrm{C}$ predicted by Ericksen's TIF model, Eqs. (15) and (19). We see oscillations in the stress or apparent viscosity, which eventually dampen out at increased strains $(\gamma \approx 200)$. We also see doublet peaks caused by the faster rotation of the director as it passes through the vertical position (Hinch and Leal 1973; Gu and Jamieson 1994). The strain period is extracted from these curves, and using Eq. (18), the tumbling parameter is calculated. $8 \mathrm{CB}$ was found to tumble for temperatures ranging form 34.2 to $38.4^{\circ} \mathrm{C}$ and flow-align at all higher temperatures up to the nematicisotropic transition. The tumbling parameters measured by this method are shown in Fig. 12, along with tumbling parameters determined from the individual Leslie viscosities reported in the literature (Kneppe et al. 1982, 1981; $\mathrm{Gu}$ and Jamieson 1994) for comparison. As can be seen in Fig. 12, our measured $\lambda$ values agree well with those obtained by Gu and Jamieson, using this same method, as well as those of Kneppe et al., in which the individual viscosities were measured by other methods and then used to determine $\lambda$.

The other single-component LCs tested by this method were $\mathrm{PCH}-5$ and $7 \mathrm{CB}$. $\mathrm{PCH}-5$ was found to flow-align throughout its entire nematic range from $30.2{ }^{\circ} \mathrm{C}$ up to $55.1{ }^{\circ} \mathrm{C}$, and no estimate of $\lambda$ was obtained. 7CB was found to have a transition from tumbling to flow-aligning somewhere between $32.3^{\circ} \mathrm{C}$ and $33.2^{\circ} \mathrm{C}$. Unlike the other tumbling nematic, $8 \mathrm{CB}$, the strain periods for $7 \mathrm{CB}$ were very large (between 57 and 127 strain units), leading to tumbling parameters very near 1 , ranging from $\lambda=0.994$ at $29.0^{\circ} \mathrm{C}$ to $\lambda=0.999$ at $33.2^{\circ} \mathrm{C}$. Typical tumbling responses for $7 \mathrm{CB}$ are shown in Fig. 13 to demonstrate much longer

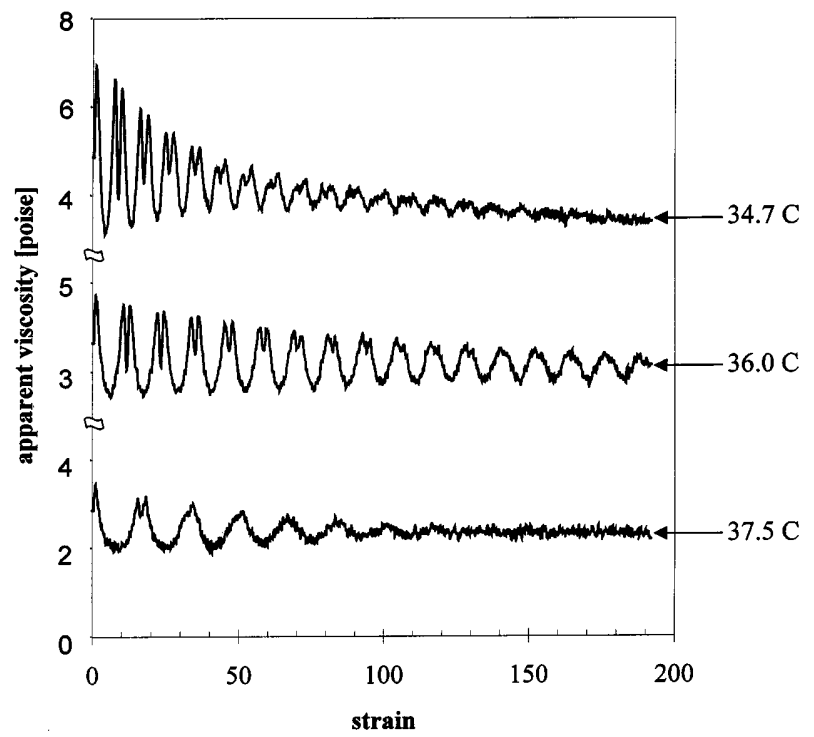

Fig. 11 Apparent viscosity versus strain for $8 \mathrm{CB}$ on shear start-up at $16 \mathrm{~s}^{-1}$ for three temperatures: $34.7,36.0$, and $37.5^{\circ} \mathrm{C}$. (Note: curves are shifted for clarity) 


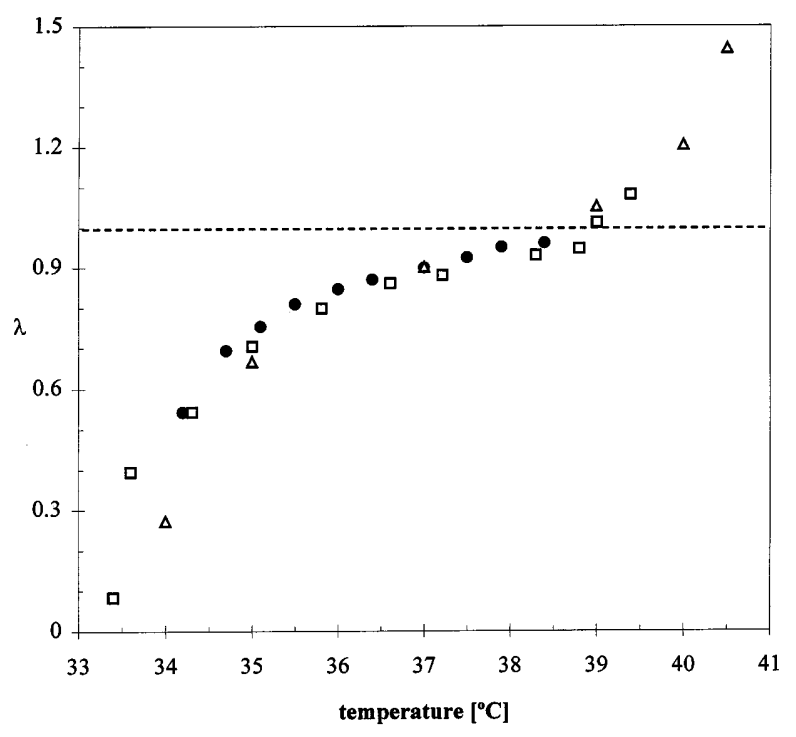

Fig. 12 Measured $\lambda$ versus temperature for $8 \mathrm{CB}$ as determined by the rheological method $(\mathbf{0})$. Results of $\mathrm{Gu}$ and Jamieson (1994) ( $\square$ ) obtained by the same method and also Kneppe's $(1982,1981)(\Delta)$ results obtained by measuring the individual Leslie viscosities are shown for comparison

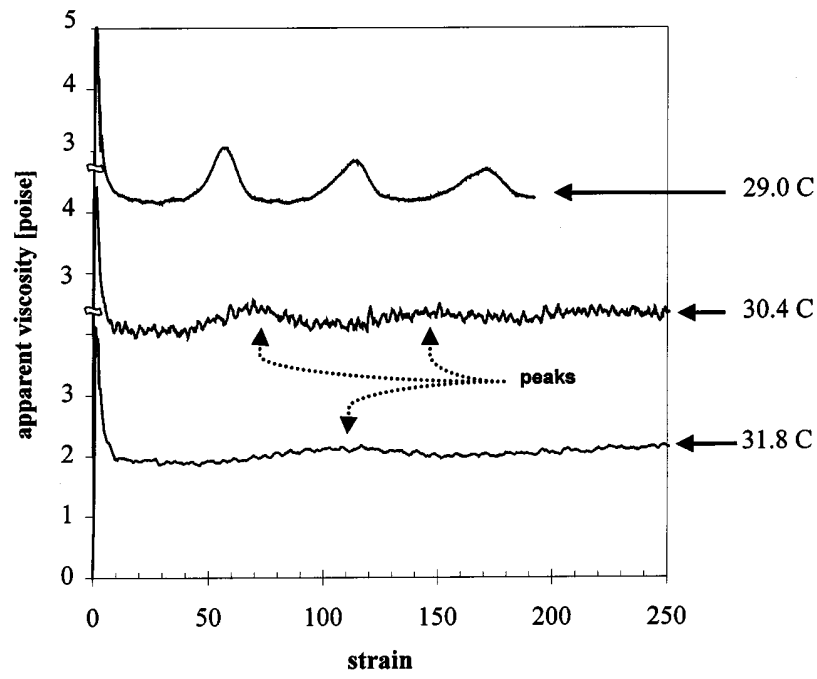

Fig. 13 Apparent viscosity versus strain for $7 \mathrm{CB}$ on shear start-up for three temperatures: $29.0,30.4$, and $31.8^{\circ} \mathrm{C}$. Curves for 29.0 and $30.4^{\circ} \mathrm{C}$ obtained with shear rate equal to $16 \mathrm{~s}^{-1}$, while the run at $31.8^{\circ} \mathrm{C}$ was obtained at $32 \mathrm{~s}^{-1}$. (Note: curves are shifted for clarity)

oscillation cycles than were present in the $8 \mathrm{CB}$ results shown in Fig. 11 . At $33.2{ }^{\circ} \mathrm{C}$ and above, $7 \mathrm{CB}$ was found to flow-align and no estimate of $\lambda$ was obtained. A comparison between the tumbling parameter obtained by this method and that obtained by the MPB method described earlier can be seen in Fig. 6 . Within experimental error, the values agree quite well between the two methods.
Given the rather anomalous results obtained by the MPB method for the commercial mixtures presented in Fig. 9, and the qualitative nature of the results obtainable from the direct method, the commercial mixtures (E7, ZLI-3449-100, and ZLI-3308) were tested by the rheological method to check for consistency with the other methods. Since no cooling was used on the rheometer, the lowest attainable temperature was around $25^{\circ} \mathrm{C}$, while the highest temperature accessible experimentally was around $95^{\circ} \mathrm{C}$. Since these mixtures have very low transition temperatures to the smectic or crystalline phase and the last mixture, ZLI-3308, has a very high clearing point, our study is limited to their nematic range of 25$95^{\circ} \mathrm{C}$. In contrast to the results of the MPB method for these materials, it was found that these three commercial mixtures flow-align throughout their entire accessible nematic range, $25-95^{\circ} \mathrm{C}$.

In partial summary of the results thus far, we have found that the single-component LCs studied here agree in the qualitative distinction between tumbling and flow-aligning behavior among all four methods. The multi-component mixtures, however, are inconsistent in that the MPB method predicts that the mixtures, E7, ZLI-3449-100, and ZLI-3308, tumble throughout most of their nematic temperature range, while the other three methods all indicate that they flow-align.

As a first step towards trying to understand the unusual behavior of these multi-component commercial mixtures, and particularly why we see this disagreement in the prediction of $\lambda$ for the four methods, we studied a couple of binary mixtures. The first binary mixture studied was the mixture of the nematic, $7 \mathrm{CB}$, with the nematic, $8 \mathrm{CB}$. The results shown earlier for this method show that pure $7 \mathrm{CB}$ and $8 \mathrm{CB}$ each tumble throughout most of their nematic phase, with flow-aligning behavior seen only very close to their respective nematic-isotropic transition temperatures. The phase behavior of the mixture, $7 \mathrm{CB} / 8 \mathrm{CB}$, was determined by $\mathrm{DSC}$ and is given in Fig. 1. Three different mixtures were made and tested, 25,50 and $75 \%$ weight fractions of $7 \mathrm{CB}$ and $8 \mathrm{CB}$. The measured tumbling parameters for these mixtures are given in Fig. 14, where Fig. 14a shows the tumbling parameter plotted against temperature for the three mixtures as well as the pure components and Fig. 14b shows the data plotted against concentration as interpolated from the data of Fig. 14a. All three mixtures were found to tumble at the lower temperatures in their nematic range, with the transition from tumbling to flow-aligning shifted to higher temperatures for the mixtures containing a higher fraction of $8 \mathrm{CB}$. Figure $14 \mathrm{~b}$ reveals a bowed shape, with the tumbling parameter shifted higher than would be expected by the simple linear relationship measured by the MPB method for this mixture, also shown in Fig. 7. Lines in 
Fig. 14b, indicating the best fits obtained using the continuum theory of Rey (1996), reveal that the data is fit well by Eq. (4) if $R$ is assumed to be independent of concentration and also allowed to vary as a fitting parameter in each case.

The other binary mixture was that of a flow-aligning nematic, $5 \mathrm{CB}$, with a tumbling nematic, $8 \mathrm{CB}$. The phase behavior of this mixture was obtained by hot-stage polarizing microscopy and is shown in Fig. 2. Again, three different mixtures were tested, 25, 50, and $75 \%$ weight fractions of $5 \mathrm{CB}$ and $8 \mathrm{CB}$. The mixtures containing $50 \%$ and $75 \% 5 \mathrm{CB}$ were found to flow-align throughout their entire nematic range, while the $25 \%$ mixture showed a transition from tumbling to flowaligning at a temperature between $33.2{ }^{\circ} \mathrm{C}$ and $34.0^{\circ} \mathrm{C}$. The strain periods were all quite high, and the corresponding tumbling parameters varied from $\lambda=0.927$ at $25.8^{\circ} \mathrm{C}$ up to $\lambda=0.998$ at $33.2^{\circ} \mathrm{C}$. Figure 15 a shows the tumbling parameter measured for the mixture containing $25 \% 5 \mathrm{CB}$ by weight, as well as that of pure $8 \mathrm{CB}$ for comparison. Since $5 \mathrm{CB}$ is a flow-aligning nematic and $8 \mathrm{CB}$ is a tumbling nematic we find that dissolving a modest amount of a flow-aligning nematic, $\sim 25 \%$, converts a tumbling nematic into a flow-aligning nematic or at least reduces the rate of tumbling significantly.

Remarkably, the measurements of the MPB method for all the pure $\mathrm{LCs}, 5 \mathrm{CB}, 7 \mathrm{CB}$, and $8 \mathrm{CB}$, agree well with the results of the rheological measurements (i.e., the open circles in Figs. 14b and $15 \mathrm{~b}$ are close to the filled diamonds for volume fractions 0 and 1 of $8 \mathrm{CB}$ ). For all the mixtures, $7 \mathrm{CB} / 8 \mathrm{CB}, 5 \mathrm{CB} / 8 \mathrm{CB}$, E7, ZLI-3449-100, and ZLI-3308, however, the MPB method gives distinctly lower values of $\lambda$ than are obtained by the other methods. This implies either an anomalously high degree of experimental error in the MPB method for these mixtures, or more likely a breakdown of the single director Leslie-Ericksen theory for mixtures of LCs. Since the results of the rheological method also agree with those of the two qualitative methods, the direct method and the twist wall method, we feel that the rheological method more accurately reflects the tumbling behavior of mixtures. Although it is true that applying the single director Leslie-Ericksen theory to mixtures is likely to be at fault for all methods used, it appears that the discrepancy may be more pronounced in the MPB method (with homeotropic boundary conditions), where the measurement of $\lambda$ relies on very small oscillations of the director far from the flowalignment angle.

A possible explanation for the apparent failure of the Leslie-Ericksen theory that we have observed in LC mixtures is suggested by a recent theory of Fialkowski (1997, 1998). He showed that in uniaxial nematics composed of biaxial molecules there are contributions to the stress arising from shear-induced biaxiality that are not included in the Leslie-Ericksen theory. If such contributions are especially large in mixtures, and if they affect the rotation rate of the director such that in the homeotropic alignment the apparent value of $\lambda$ is lower than at other orientations, this might account for the
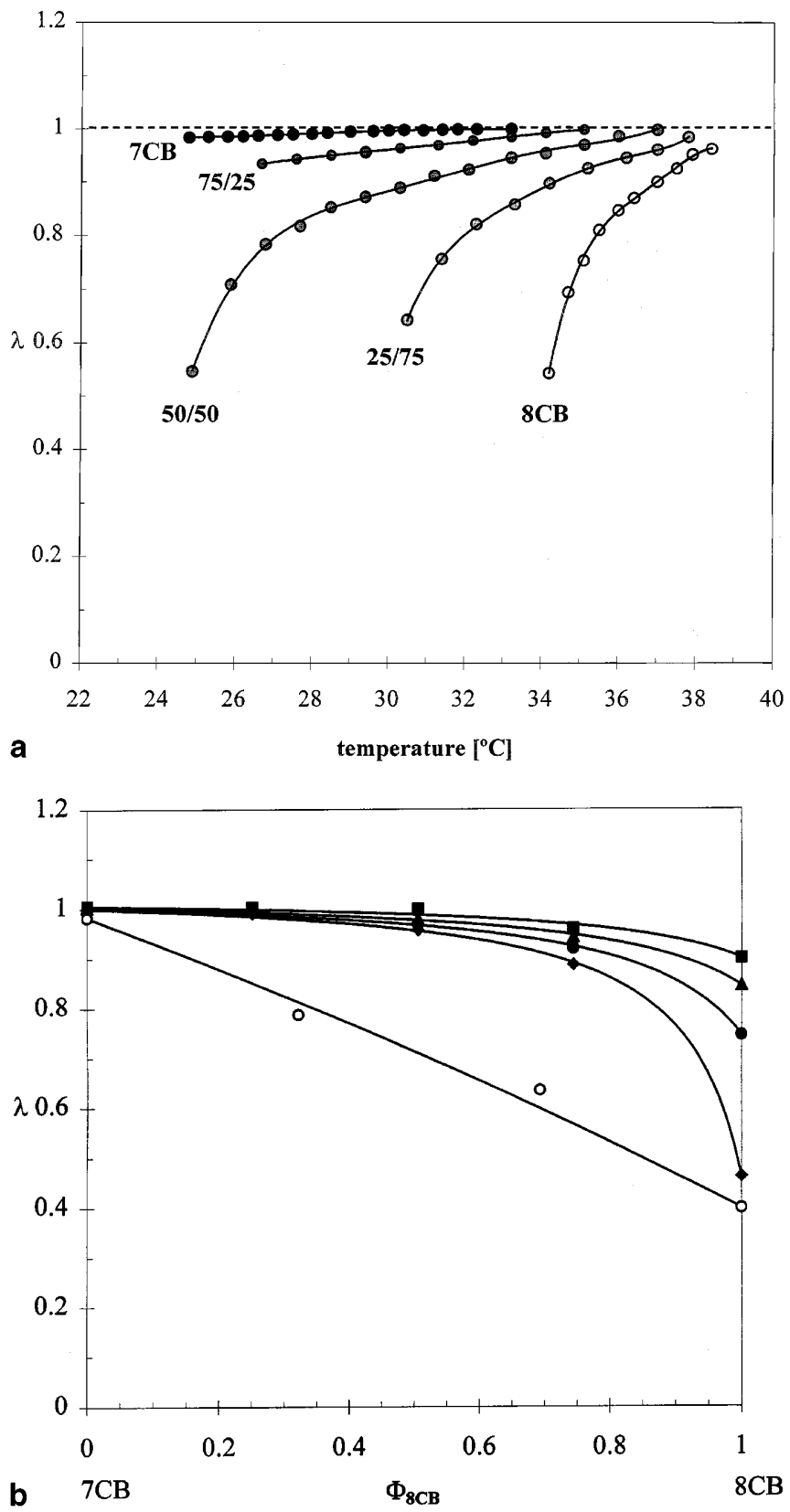

Fig. 14a, b Results for $7 \mathrm{CB} / 8 \mathrm{CB}$ mixtures as obtained by the rheological method. a $\lambda$ versus $T$ for the pure components $7 \mathrm{CB}$ and $8 \mathrm{CB}$, and three mixtures: 25,50 , and $75 \%$ by weight. b $\lambda$ versus volume fraction interpolated from Fig. 14A for four temperatures: $34^{\circ} \mathrm{C}(\diamond), 35^{\circ} \mathrm{C}(\boldsymbol{\bullet}), 36^{\circ} \mathrm{C}(\boldsymbol{\Delta})$, and $37^{\circ} \mathrm{C}(\boldsymbol{\square})$. Results obtained by the MPB method at $33.6^{\circ} \mathrm{C}(\mathrm{O})$ are also shown for comparison. Lines showing best obtainable fit by the phenomenological theory of Rey are shown with $R$ values in each case: $R=0.087(\diamond), R=0.146(\bullet)$, $R=0.183(\mathbf{\Delta}), R=0.193($ 

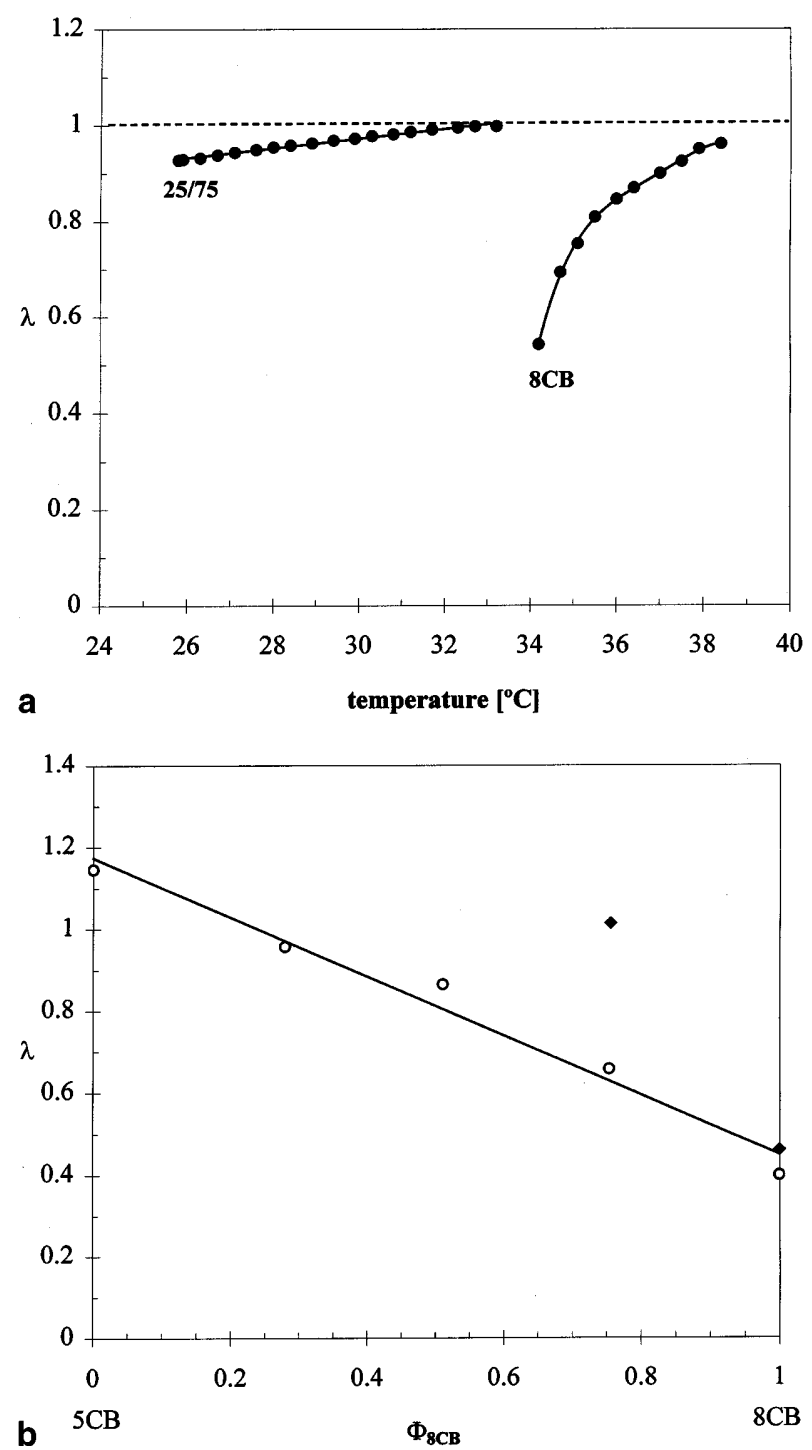

Fig. 15a, b Results for $5 \mathrm{CB} / 8 \mathrm{CB}$ mixtures as obtained by the rheological method. a $\lambda$ versus $T$ for the pure component $8 \mathrm{CB}$ and the $25 / 75$ mixture of $5 \mathrm{CB} / 8 \mathrm{CB}$. Lines for pure $5 \mathrm{CB}, 50 / 50$, and $75 / 25$ mixtures are not shown since for these mixtures $\lambda>1$ and its value is not measurable by this method. b $\lambda$ versus volume fraction interpolated from plot $1 \mathrm{a}$ for $33.6^{\circ} \mathrm{C}(\diamond)$. Results obtained by the MPB method at $33.6^{\circ} \mathrm{C}(\bigcirc)$ are also shown for comparison. Line shown to aid the eye only and represents the best straight line fit anomalously small value of $\lambda$ obtained by the MPB method, since in the MPB method the director never rotates very far from homeotropic alignment.

\section{Conclusions}

In this work, the tumbling parameter, $\lambda$, is measured for various small-molecule LCs and their mixtures. The methods used include textural observations (twist walls), a direct method, the oscillatory method developed by Mather, Pearson, and Burghardt (1995), and a rheological method. Comparisons are made for each single-component system with a molecular theory by plotting $\lambda$ versus the reduced temperature, $T_{\mathrm{r}}=T / T_{\mathrm{ni}}$. For the binary mixtures, the continuum theory of Rey (1996) is found to fit the data well provided $R$ is concentration independent and allowed to vary as a fitting parameter in each case. For single-component small-molecule LCs, the four methods are found to be consistent with each other in their predictions of $\lambda$, and the temperature dependence of the tumbling parameter agrees quite well with the molecular theory of Archer and Larson (1995). For the multi-component mixtures studied here, however, the four methods are not consistent with each other in predicting $\lambda$. Specifically, the oscillatory method of MPB underpredicts the tumbling parameter in all cases. This suggests a breakdown in the single director Leslie-Ericksen theory when applied to mixtures of LCs.

Acknowledgement This work was supported by the MRL Program of the National Science Foundation under Award No. DMR9632716.

\section{References}

Abdoh MMM, Srinivasa, Shivaprakash NC, Prasad S (1982) Orientational order in the nematogenic homologous series trans-4-alkyl(4-cyanophenyl)cyclohexane. J Chem Phys 77:25702576

Archer LA, Larson RG (1995) A molecular theory of flow alignment and tumbling in sheared nematic liquid crystals. J Chem Phys 103:3108-3111
Becker ME, Kilian RA, Kosmowski BB, Mlynski DA (1986) Alignment properties of rubbed polymer surfaces. Mol Cryst Liq Cryst 132:167-180

Born M, Wolf E (1980) Principles of optics, 6th edn. Pergamon Press, Oxford

Bruinsma RF, Safinya CR (1991) Landau theory of the nematic-smectic-A phase transition under shear flow. Phys Rev A 43:5377-5404
Burghardt WR, Fuller GG (1991) Role of director tumbling in the rheology of polymer liquid crystal solutions. Macromolecules 24:2546-2555

Carlsson T (1984) Theoretical investigation of the shear flow of nematic liquid crystals with the Leslie viscosity $\alpha_{3}>0$ : hydrodynamic analog of first order phase transitions. Mol Cryst Liq Cryst 104:307- 334 
Carlsson T, Skarp K (1986) Observation of the tumbling instability in torsional shear flow of a nematic liquid crystal with $\alpha_{3}>0$. Liq Cryst 1:455-471

Ericksen JL (1960) Anisotropic fluids. Arch Rat Mech Anal 4:231-237

Fialkowski M (1997) Viscous properties of biaxial nematic liquid crystals: the method of calculation of the Leslie viscosity coefficients. Phys Rev E 55:2902-2915

Fialkowski M (1998) Viscous properties of nematic liquid crystals composed of biaxial molecules. Phys Rev 58:19551966

Gähwiller C (1972) Temperature dependence of flow alignment in nematic liquid crystals. Phys Rev Lett 28:1554 1556

Graf H-H, Kneppe H, Schneider F (1992) Shear and rotational viscosity coefficients of two nematic liquid crystals. Mol Phys 77:521-538

Gu D-F, Jamieson AM, Wang S-O (1993) Rheological characterization of director tumbling induced in a flow-aligning nematic solvent by dissolution of a side-chain liquid-crystal polymer. J Rheol 37:985-1001

Gu D-F, Jamieson AM (1994) Shear deformation of hometropic monodomains: temperature dependence of stress response for flow-aligning and tumbling nematics. J Rheol 38:555-571

Hartshorne NH, Stuart A (1970) Crystals and the polarizing microscope, 4 th edn. Arnold, London

Hinch EJ, Leal LG (1973) Time-dependent shear flows of a suspension of particles with weak Browninan rotations. J Fluid Mech 57:753-767

Karat PP, Madhusudana NV (1976) Elastic and optical properties of some $4^{\prime}-n$ alkyl-4-cyanobiphenyls. Mol Cryst Liq Cryst 36:51-64

Kneppe H, Schneider F (1981) Determination of the viscosity coefficients of the liquid crystal MBBA. Mol Cryst Liq Cryst 65:23-38

Kneppe H, Schneider F, Sharma NK (1981) A comparative study of the viscosity coefficients of some nematic liquid crystals. Ber Bunsenges Phys Chem 85:784-789

Kneppe H, Schneider F, Sharma NK(1982) Rotational viscosity $\gamma_{1}$ of nematic liquid crystals. J Chem Phys 77:32033208

Kröger M, Sellers HS (1995) Viscosity coefficients for anisotropic, nematic fluids based on structural theories of suspensions. J Chem Phys 103:807817

Kuzuu N, Doi M (1983) Constitutive equation for nematic liquid crystals under weak velocity gradient derived from a molecular kinetic equation. J Phys Soc Jpn 52:3486-3494

Kuzuu N, Doi M (1984) Constitutive equation for nematic liquid crystals under weak velocity gradient derived from a molecular kinetic equation. II Leslie coefficients for rodlike polymers. J Phys Soc Jpn 53:1031-1040

Larson RG (1993) Roll-cell instabilities in shearing flows of nematic polymers. J Rheol 37:175-197

Lide DR (1994) CRC handbook of chemistry and physics, 75th edn CRC Press, London

Magda JJ, Baek S-G, DeVries KL, Larson RG (1991) Shear flows of liquid crystal polymers-measurements of the 2nd normal stress difference and the Doi molecular theory. Macromolecules 24:4460-4468

Maier W, Saupe AZ (1959) Eine einfache molekular-statistische theorie der nematischen kristallin-flüssigen phase. Teil I. Z Naturforsch A14:882-889

Manneville P (1976) Shear flow instability in nematic liquids. Theory. Steady simple shear flows. J Phys 37:285-96
Manneville P (1981) The transition to turbulence in nematic liquid crystals. Mol Cryst Liq Cryst 70:1501-1528

Mather PT (1994) Ph.D. dissertation, University of California, Santa Barbara

Mather PT, Pearson DS, Burghard WR (1995) Structural response of nematic liquid crystals to weak transient shear flows. J Rheol 39:627-648

Mather PT, Pearson DS, Larson RG, Gu D-F, Jamieson AM (1997) The origin of stress-oscillation damping during start-up and reversal of torsional shearing of nematics. Rheol Acta 36:485497

Mosley A, Nicholas BM, Gass PA (1987) Surface alignment of liquid crystals by rubbed polymer layers. Disp Technol Appl 8:17-21

Pieranski P, Guyon E (1974) Instability of certain shear flows in nematic liquids. Phys Rev A 9:404-417

Rey AD (1995) Phenomenological theory of flow-alignment in binary nematic mixtures. Rheol Acta 34:461-473

Rey AD (1996) Flow-alignment and viscosity rules for single-phase binary mesomorphic mixtures. Liq Cryst 20:147-159

Scheffer TJ, Nehring J (1977) Accurate determination of liquid-crystal tilt bias angles. J Appl Phys 48:1782-1792

Sirinivasarao M, Berry GC (1991) Rheooptical studies on aligned nematic solutions of a rodlike polymer. J Rheol 35:379-397

Viola GG, Baird DG (1986) Studies on the transient shear flow behavior of liquid crystalline polymers. J Rheol 30:601628

Yang I-K, Shine AD (1993) Transient shear flow of a unidomain liquid crystalline polymer. Macro-molecules 26:15291536

Zuñiga I, Leslie FM (1989) Shear-flow instabilities in non-flow-aligning nematic liquid crystals. Liq Cryst 5:725-734 